\title{
Variable Equation of State for Generalised Dark Energy Model
}

\author{
Saibal Ray* \\ Department of Physics, Government College of Engineering ES Ceramic Technology, Kolkata 700 010, West Bengal, India \\ Farook Rahaman ${ }^{\dagger}$ \\ Department of Mathematics, Jadavpur University, Kolkata 700 032, West Bengal, India \\ Utpal Mukhopadhyay \\ Satyabharati Vidyapith, Barasat, North 24 Parganas, Kolkata 700 126, West Bengal, India \\ Ruby Sarkar ${ }^{\S}$ \\ Future Campus School, Sonarpur Station Road, Kolkata 700 150, West Bengal, India
}

(Dated: August 8, 2018)

\begin{abstract}
We present a model for the present accelerating Universe and focus on the different important physical variables involved in the model under the phenomenological assumption $\Lambda \propto H^{2}$ with a prescription for equation of state parameter in the form $\omega(t)=\omega_{0}+\frac{\omega_{1} \tau}{t^{2}}$, where $\omega_{0}$ and $\omega_{1}$ are two constants and $\tau$ is a parameter having dimension of time $t^{2}$. General expressions for the density parameter $\Omega$ and deceleration parameter $q$ are obtained which under specific bound reproduce some of the previous results. We explore physical features of these parameters which (i) provide the scenario of complete evolution of the cosmos with $\omega(t)$ and (ii) agree mostly with the observational status of the present phase of the accelerating Universe.

PACS numbers: 04.20.Jb, 98.80.Jk, 98.80.Cq.
\end{abstract}

\section{INTRODUCTION}

Ever since the discovery of an Universal acceleration [1, 2], great efforts are going on for finding a satisfactory explanation of this effect. Most of the works related to this problem are based on the assumption that an exotic type of energy, termed as dark energy, is the causative agent of this acceleration although alternative explanations are also available in the literature [3 5$]$. Phenomenological models are also contenders in this race for understanding the elusive dark energy. A number of phenomenological models involving time-dependent cosmological term $\Lambda$ have already been proposed and tested by various workers from different standpoints (for an overview see Overduin and Cooperstock [6] and Sahni and Starobinsky [7]). In favor of $\Lambda$-decay scenario, irrespective of whether they come from extended theories of gravity or phenomenological considerations, it is argued [6, 8] that (i) they have been shown to address a number of pressing problems in cosmology; (ii) many are independently motivated, e.g., by dimensional arguments, or as limiting cases of more complicated theories; (iii) most are simple enough that meaningful conclusions can be drawn about their viability and (iv) successful implementation would point either towards the eventual Lagrangian formulation or a more complete theory.

\footnotetext{
*saibal@iucaa.ernet.in

†farook_rahaman@yahoo.com

${ }^{\ddagger}$ utpal1739@gmail.com

§rubyfantacy@yahoo.co.in
}

A very important parameter for the above dark energy investigation is that of the equation of state parameter $\omega$. For pressureless dust, radiation, stiff-fluid and vacuum-fluid dominated Universe, one can assume the values $0,1 / 3,1$ and -1 respectively for $\omega$. Some other limits obtained from observational results coming from SN Ia data 9] and SN Ia data corroborated with CMBR anisotropy and galaxy clustering statistics [10] are $-1.67<\omega<-0.62$ and $-1.33<\omega<-0.79$ respectively. However, it is not at all obligatory to use a constant value of $\omega$. In fact, it can be a function of time, redshift or scale factor [11-13]. But, due to lack of conclusive observational data which may enable us to distinguish between a variable and constant equation of state, usually a constant value of $\omega$ is used [14, 15]. Coming to the case of particular relationship of variable $\omega$ with redshift, it can be linear like $\omega(z)=\omega_{0}+\omega^{\prime} z$ where $\omega^{\prime}=(d \omega / d z)_{z=0}$ [16, 17] or non-linear as $\omega(z)=\omega_{0}+\omega_{1} z /(1+z)$ [18, 19]. So, as far as the scale factor dependence of $\omega$ is concerned, the parametrization $\omega(a)=\omega_{0}+\omega_{a}(1-a)$, where $\omega_{0}$ is the present $(a=1)$ value and $\omega_{a}$ is a measure of the time variation $\omega^{\prime}$, is widely used in the literature [20]. Different forms of $\omega_{a}$ are also available in various works [19, 21]. Some other models such as kink model 22] and e-fold model [23] have tried to construct a monotonic $\omega(a)$ involving four parameters which makes transition from some asymptotic past value $\omega_{p}$ to some asymptotic future value $\omega_{f}$. Physical supports in favor of $\omega_{a}$ parametrization have been given by Linder [19, 24]. In quintessence models involving scalar fields give rise to time-dependent $\omega$ 25 29]. There are also some cosmological models with a time dependent equation of state parameter available in the literature with Kaluza-Klein 
metric and wormholes 30 32].

Another aspect of the accelerating Universe is related with the signature flipping of the deceleration parameter $q$. In fact, in the present dark energy dominated Universe, the second largest constituent is another dark component, viz. dark matter. About 25\% contribution from the matter content of the Universe is in the form of dark matter. Now, in an ever accelerating Universe, hierarchical structure formation is impossible. A primary criteria, therefore, for the $\Lambda$-CDM Universe is that the present acceleration is a recent phenomena and was preceded by a period of deceleration. Hence the deceleration parameter $q$ must have changed its sign sometime during cosmic evolution. Both theoreticai [33, 34] and observational [35] works support this physical idea. So, $q$ must be time-dependent so that the transition of the Universe from a decelerating to an accelerating phase can occur smoothly. This point must be kept in mind while dealing with dark energy investigation regarding present accelerating Universe.

In recent years various forms of time-dependent $\omega$ have been used for variable $\Lambda$ models, viz. $\dot{\Lambda} \propto H^{3}$, [36 38], $\Lambda \propto \dot{H}$ [65] and $\Lambda \propto H^{2}$ [40]. Recently Ray et al. [41] have shown the equivalence of the three $\Lambda$ models, viz. $\Lambda \propto H^{2}, \Lambda \propto \ddot{a} / a$ and $\Lambda \propto \rho$ for constant $\omega$. It was mentioned in that work of Ray et al.[41] that in a later work, a more accurate analysis may be made by considering a time-varying equation of state parameter whereas Mukhopadhyay et al. [40] have already investigated the $\Lambda \propto H^{2}$ model for a particular type of variable $\omega$ with the full physical range of $\alpha$. So, the purpose of the present paper is to re-examine the status of the same $\Lambda \propto H^{2}$ model for the presciption $\omega(t)=\omega_{0}+\frac{\omega_{1} \tau}{t^{2}}$, where $\omega_{0}$ and $\omega_{1}$ are two constants and $\tau$ is a parameter having dimension of $t^{2}$. The scheme of the investigation is as follows: In the Section 2 the Einstein field equations and their solutions are provided whereas Section 3 deals with the physical features of different parameters. In Section 4 some comments are made on the basis of the results obtained in the investigations.

\section{THE EINSTEIN FIELD EQUATIONS AND THEIR SOLUTIONS}

The Einstein field equations are

$$
R^{i j}-\frac{1}{2} R g^{i j}=-8 \pi G\left[T^{i j}-\frac{\Lambda}{8 \pi G} g^{i j}\right],
$$

where the erstwhile cosmological constant $\Lambda$ is assumed as a function of time, viz. $\Lambda=\Lambda(t)$ and the velocity of light $c$ in vacuum is unity when expressed in relativistic units.

In this connection it is to be mention that invariant property of $\Lambda$ under Lorentz transformation is not satisfied for arbitrary systems (e.g., material systems and radiation). It has been argued that the energy density of vacuum represents a scalar function of the four- dimensional space-time coordinates so that it satisfies the Lorentz symmetry [42, 43]. On the other hand Vishwakarma [44] considered a particular Ricci-symmetry under the framework of general relativity which is the contracted Ricci-collineation along the fluid flow vector and argues that this symmetry does demand Lambda to be a function of time (and space, in general). In this context we would also like to mention that by the application of renormalization group approach of quantum field theory in cosmology Shapiro et al. 45] have derived generalized energy conservation laws for variable $\Lambda$ models. Such generalized laws have also been deduced by Vereschagin et al. [46] based on the work of Gurzadyan and Xue [47]. However, comment made by Beesham [48] and Shapiro et al. [45] that for simplicity, one may still retain the usual energy conservation law even when $\Lambda$ is a variable. In the present investigation, therefore, we have used the same energy conservation law for solving the dynamical cosmological equation.

For the spherically symmetric Friedmann-LemaitreRobertson-Walker (FLRW) metric

$$
d s^{2}=-d t^{2}+a(t)^{2}\left[\frac{d r^{2}}{1-k r^{2}}+r^{2}\left(d \theta^{2}+\sin ^{2} \theta d \phi^{2}\right)\right],
$$

where $a$ is the scale factor and the curvature constant $k$ is $-1,0,+1$ respectively for open, flat and close models of the Universe, the Einstein field equations (1) take the forms as

$$
\begin{aligned}
& \left(\frac{\dot{a}}{a}\right)^{2}+\frac{k}{a^{2}}=\frac{8 \pi G}{3} \rho+\frac{\Lambda}{3}, \\
& \frac{\ddot{a}}{a}=-\frac{4 \pi G}{3}(\rho+3 p)+\frac{\Lambda}{3} .
\end{aligned}
$$

From equations (3) and (4), one can arrive at the equation [4]

$$
\left(\frac{\dot{a}}{a}\right)^{2}+\left[3\left(\frac{1+w}{1+3 w}\right)-1\right] \frac{\ddot{a}}{a}+\frac{k}{a^{2}}=\left(\frac{1+w}{1+3 w}\right) \Lambda,(5)
$$

where the time-dependent barotropic equation of state parameter $\omega(t)$ is given by $\omega(t)=p(t) / \rho(t)$.

Let us use the ansatz $\Lambda=3 \alpha H^{2}$. This is one of the very well known and widely used phenomenological models as proposed by Carvalho et al. [49] and Waga [50] from dimensional arguments. However, Lima and Carvalho [51] have considered the same model from different point of view. According to Vishwakarma [52], one can obtain the relationship $\Lambda \propto H^{2}$ when the cosmological term $\Lambda$ is expressed in terms of Planck energy density. Moreover, it has been demonstrated by other authors [53, 54 that $\Lambda \propto H^{2}$ law can also be deduced by using effective field theory and black hole thermodynamics.

By the use of this ansatz $\Lambda=3 \alpha H^{2}$ in the equation (5)), we arrive at the following form

$$
H^{2}+\frac{2}{(1+3 \omega)}\left(\dot{H}+H^{2}\right)=\frac{1+\omega}{(1+3 \omega)} 3 \alpha H^{2}
$$


which, on simplification becomes

$$
\frac{d H}{H^{2}}=-\frac{3(1-\alpha)}{2}(1+\omega) d t .
$$

Now, it is important to consider a suitable form of the equation of state parameter $\omega$. As already mentioned in the Introduction that it can be time-dependent and even it may be a function of the red-shift $z$ or scale factor $a$ as well. For time-dependence Mukhopadhyay et al. [55] suggest it in the form $\omega(t)=\omega_{0}+\omega_{1} t^{n}$ which can be regarded as a generalization of the special form $\omega(t)=$ $\omega_{0}+\omega_{1} t$ [36]. In the present work, let us suppose the structure of $\omega$ as follows

$$
\omega(t)=\omega_{0}+\frac{\omega_{1} \tau}{t^{2}},
$$

where $\omega_{0}$ and $\omega_{1}$ are two constants and $\tau$ is a parameter having dimension of $t^{2}$. Here $\tau$ apart from being a non-negative mathematical parameter, rather bears some deeper physical significance. It has been shown that $\tau$ represents the time-scale of evaporation of Bose-Einstein condensates which include a time-dependent $\Lambda$ [56 58 ]. In this context it is interesting to note that Mukhopadhyay et al. 55 consider $\tau$ in their equation of state in the form $\tau=t /(1+\omega)^{1 / n}$. Thus, they argue that for the present dust-filled Universe $(\omega=0), \tau$ is equal to $t$ whereas for vacuum fluid $\tau$ becomes meaningless. However, in the present case the expression for $\omega$ immediately suggests that for very small $t$, i.e. in the early Universe, the contributions from the second term, $\omega_{1} \tau / t^{2}$, in the right hand side of equation (8) were significant. But for large $t$, i.e. for late Universe, this term tends to zero and consequently $\omega(t)$ converges to a nearly constant value $\omega_{0}$. This may be one of the reasons that at present we can not distinguish between a constant and a variable equation of state as mentioned in the Introduction.

By the use of the equation (8) in equation (7) and solving the resulting equation we get our solution set as

$$
\begin{gathered}
a(t)=\left[\left(1+\omega_{0}\right) t^{2}-\omega_{1} \tau\right]^{\frac{1}{3(1-\alpha)\left(1+\omega_{0}\right)}}, \\
H(t)=\frac{2 t}{3(1-\alpha)\left[\left(1+\omega_{0}\right) t^{2}-\omega_{1} \tau\right]}, \\
\rho(t)=\frac{t^{2}}{6 \pi G(1-\alpha)\left[\left(1+\omega_{0}\right) t^{2}-\omega_{1} \tau\right]^{2}}, \\
\Lambda(t)=\frac{4 \alpha t^{2}}{3(1-\alpha)\left[\left(1+\omega_{0}\right) t^{2}-\omega_{1} \tau\right]^{2}} .
\end{gathered}
$$

Now, since the Universe is at present old enough, one can easily neglect the terms involving powers of $1 / t^{2}$ greater than one. Thus the equations (10)-(12) transform to

$$
H(t)=\frac{2}{3(1-\alpha)\left(1+\omega_{0}\right) t}\left[1+\frac{\omega_{1} \tau}{\left(1+\omega_{0}\right) t^{2}}\right],
$$

$$
\begin{gathered}
\rho(t)=\frac{1}{6 \pi G(1-\alpha)\left(1+\omega_{0}\right)^{2} t^{2}}\left[1+\frac{2 \omega_{1} \tau}{\left(1+\omega_{0}\right) t^{2}}\right], \\
\Lambda(t)=\frac{4 \alpha}{3(1-\alpha)^{2}\left(1+\omega_{0}\right)^{2} t^{2}}\left[1+\frac{2 \omega_{1} \tau}{\left(1+\omega_{0}\right) t^{2}}\right] .
\end{gathered}
$$

From equation (13) it is clear that for large $t$, the term $\omega_{1} \tau /\left(1+\omega_{0}\right) t^{2}$ is negligibly small and hence we get the relationship $H \propto t^{-1}$. Similarly from equations (14) and (15) neglecting the terms involving $t^{-4}$ one can arrive at the relations $\rho \propto t^{-2}$ and $\Lambda \propto t^{-2}$. Thus for large $t$ we can recover the relationships $H \propto t^{-1}, \rho \propto t^{-2}$ and $\Lambda \propto t^{-2}$ obtained by Ray et al. [41] for constant $\omega$. This proves the generality of the present work so far as the time variations of $H, \rho$ and $\Lambda$ are concerned.

\section{PHYSICAL FEATURES OF THE PARAMETERS}

Using equations (13)-(15) we can obtain the expressions for the matter-energy density $\Omega_{m}$ and dark-energy density $\Omega_{\Lambda}$ as

$$
\begin{gathered}
\Omega_{m} \equiv \frac{8 \pi G \rho}{3 H^{2}}=(1-\alpha)\left[1-\frac{4 \omega_{1}^{2} \tau^{2}}{\left(1+\omega_{0}\right)^{2} t^{4}}\right], \\
\Omega_{\Lambda} \equiv \frac{\Lambda}{3 H^{2}}=\alpha\left[1-\frac{4 \omega_{1}^{2} \tau^{2}}{\left(1+\omega_{0}\right)^{2} t^{4}}\right] .
\end{gathered}
$$

Adding equations (16) and (17) we can obtain

$$
\Omega=\Omega_{m}+\Omega_{\Lambda}=1-\frac{4 \omega_{1}^{2} \tau^{2}}{\left(1+\omega_{0}\right)^{2} t^{4}} .
$$

From equations (16) and (17) we find that for large $t, \Omega_{m} \simeq(1-\alpha)$ and $\Omega_{\Lambda} \simeq \alpha$. For the same $\Lambda$ model with constant $\omega$, Ray et al. 41] obtained $\Omega_{m}=(1-\alpha)$ and $\Omega_{\Lambda}=\alpha$. So, in this case also, we find that the present work is a generalization of the work of Ray et al. [41]. Further from equation (18) we get $\Omega \simeq 1$ when $t$ is very large. This result is also compatible with the observational results and the work of Ray et al. [41]. Moreover, since the second term in the right hand side of equation (18) is clearly positive, $\Omega<1$. But, the sum of all types of energy densities being equal to 1 , the term $4 \omega_{1}^{2} \tau^{2} /\left(1+\omega_{0}\right)^{2} t^{4}$ in equation (18) can be interpreted as the sum of energy densities due to radiation and curvature, or in absence of any curvature, the energy density coming from radiation only. It has already been mentioned that for large $t$, the term $4 \omega_{1}^{2} \tau^{2} /\left(1+\omega_{0}\right)^{2} t^{4}$ is very small. This result is compatible with the idea that at present both radiation-energy density and energy density due to curvature are negligibly small and almost entire energy density of the Universe comes from matterand dark-energy densities. In this context we would like to mention the work of Mukhopadhyay et al. [59] where, 


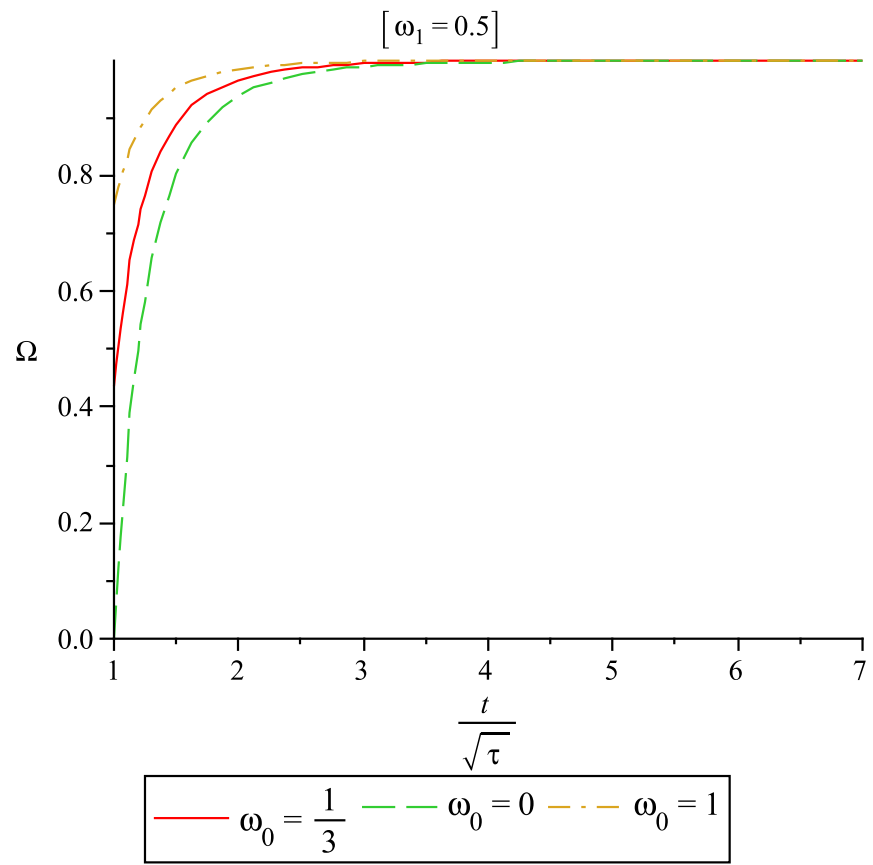

FIG. 1: Variation of density parameter with cosmic time for the specified values of $\omega_{0}$ when $\omega_{1}=0.5$ (Equation 18).

for the $\Lambda \propto H^{2}$ model with constant $\omega$, it has been shown that the sum of matter- and dark energy-densities is equal to 1 for both early and present Universe. But this result $\Omega_{m}+\Omega_{\Lambda}=1$ is valid for the late Universe also only when the Universe is composed of two fluids having barotropic indices $\omega_{a}$ and $\omega_{b}$. In the present work, however, without resorting to any special assumption, it has been possible to show that with the passage of time the density parameter $\Omega\left(=\Omega_{m}+\Omega_{\Lambda}\right)$ smoothly moves from values less than 1 to the currently accepted value 1 . This aspect is clear from the plot for $\Omega$ vs $t$ in the Fig. 1, where variation of density parameter with cosmic time for the specified values of $\omega_{0}$ when $\omega_{1}=0.5$ has been shown.

Let us now calculate the deceleration parameter to see it's physical status. For this, by using equation (10), we get the expression for the deceleration parameter $q$ as

$$
q=\frac{3(1-\alpha)}{2}\left[\left(1+\omega_{0}\right)+\frac{\omega_{1} \tau}{t^{2}}\right]-1
$$

From equation (19) we find that for small $t$, the term $\omega_{1} \tau / t^{2}$ had a significant contribution and consequently the Universe was in a decelerating phase. But with the passage of time the term $\omega_{1} \tau / t^{2}$ became negligibly small and hence the Universe switched over to the present accelerating epoch. This clearly shows the signature flipping of $q$ which is essential for the $\Lambda$-CDM cosmology. Therefore, for large $t$ one can neglect the second term inside the bracket in equation (16), when the equation

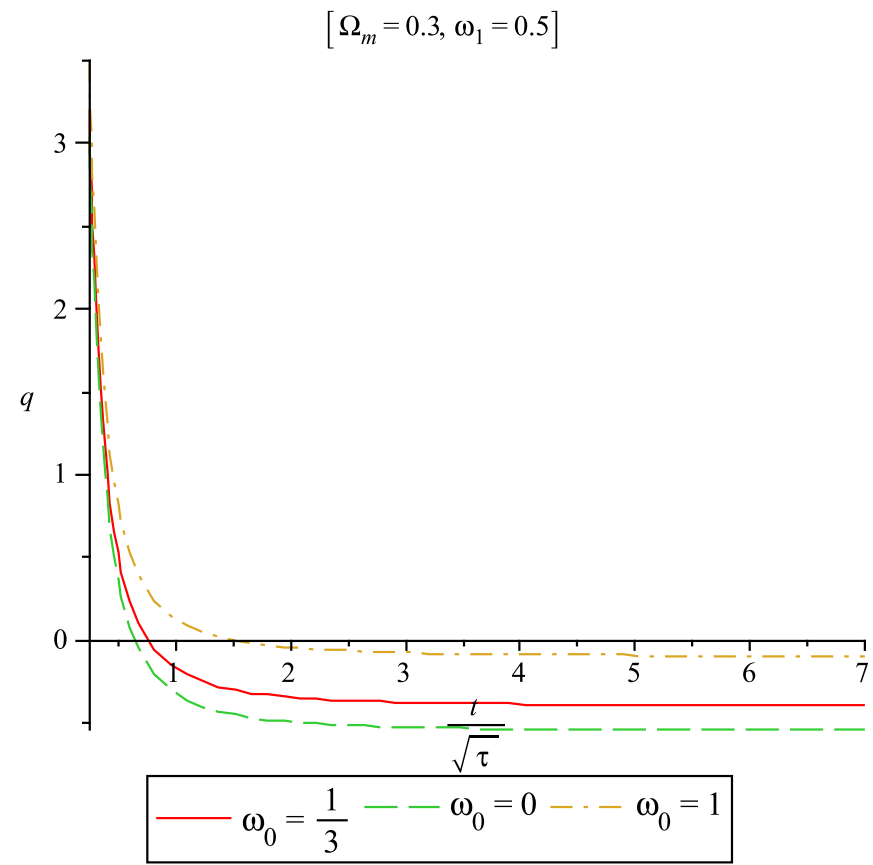

FIG. 2: Variation of deceleration parameter with cosmic time for the specified values of $\omega_{0}$ when $\omega_{m}=0.3$ and $\omega_{1}=0.5$ (Equation 20).

(19) reduces to to following form as

$$
q=\frac{3 \Omega_{m}}{2}\left[\left(1+\omega_{0}\right)+\frac{\omega_{1} \tau}{t^{2}}\right]-1
$$

Now, assuming the value $\Omega_{m}=0.30$, which is within the range $\Omega_{m}=0.330 \pm 0.035[52,6062]$ and $\omega_{0}=0$, the present value of the $q$ comes out as $q_{0}=-0.55$. This value of $q_{0}$ nicely fits with the presently accepted value of that parameter as $q_{0}=-0.50 \pm 0.05$ for an accelerating Universe 63, 64 (Fig. 2). This value of $q_{0}$ was earlier obtained by Ray et al [41] for $\omega=0$. Thus through the present work, it has been possible to recover the value of $q_{0}$ as obtained by Ray et al. [41].

In the same spirit we would like to look at the equation of state expressed in the equation (9) and plot the variation of equation of state parameter with cosmic time. The evolution of $\omega$ are shown here for radiation, dust, and stiff fluid phase with solid, dashed and chain-dashed lines respectively (Fig. 3). For all these, $\omega_{1}$ is taken to be 0.5. However, it is observed that the nature of plots related to $\omega$ does not depend on the values of $\omega_{1}$.

\section{DISCUSSION}

In the present investigation some interesting physics are explored by assuming a particular time-dependent form of the equation of state parameter $\omega$ to track down the time evolution of the Universe. It is shown that at 


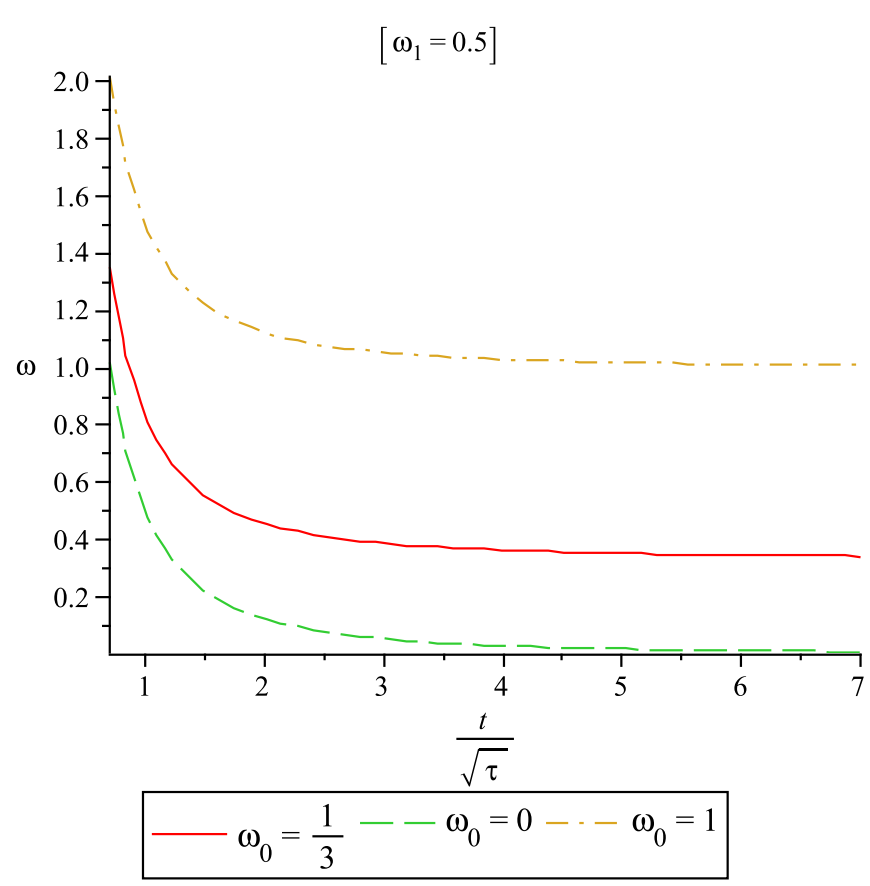

FIG. 3: Variation of equation of state parameter with cosmic time for the specified values of $\omega_{0}$ when $\omega_{1}=0.5$ (Equation 8).

present both radiation-energy density and energy density due to curvature are negligibly small such that almost the entire energy density of the Universe comes from matterand dark-energy densities. With the passage of time the cosmic density parameter $\Omega$ smoothly moves from values less than 1 to the currently accepted value 1 as shown in the Fig. 1.

It has also been possible to recover the results of Ray et al. 41] so far as the expressions of $H, \rho, \Lambda, \Omega_{m}, \Omega_{\Lambda}$ and $q$ are concerned. In particular, the expression for $q$ shows the required signature change during cosmic evolution proving that the present acceleration of the Universe is a recent phenomena (Fig. 2). The calculated value of $q$ is compatible with the modern accepted value of this parameter. The present investigation has also been interesting to show a glimpse of the small amount of radiation-energy density left over from the early Universe in the form of CMBR and tiny fraction of energy density, if it exists at all at present, due to curvature of space. This justifies the comment made by Mukhopadhyay et al. [59] that we are still not in a position to rule out $k= \pm 1$ cosmologies.

It is to note that the present work has also been successful in justifying the idea of Ray et al [41] that timedependent equation of state parameter is essential for studying the complete time-evolution of the cosmos (Fig. $3)$. In connection to the work of Mukhopadhyay and Ray 65] it is to be mentioned here that for quintessence, vacuum fluid and phantom energy, the rate of acceleration should be higher, e.g. for $\omega=-0.5,-1.0$ and -2.0 the resultant deceleration parameter values are respectively, $q=-0.775,-1.0$ and -1.45 . On the other hand, for stiff fluid $(\omega=1.0), q=-0.1$. This suggest that more smaller the value of $\omega$ the higher is the rate of acceleration resulting the so-called Big Rip [66] or Partial Rip 67] scenario due to divergence of scale factor. One can also note the work of Kuhlen et al. 68] where they consider a range of parameter space: $\omega=-0.5,-0.75,-1.0$, -1.25 and -1.5 for their simulations to reproduce the time-evolution of the Universe. However, in the present investigation by considering an equation of state in the form $\omega(t)=\omega_{0}+\frac{\omega_{1} \tau}{t^{2}}$ we are able to get the total range of time evolution of the Universe. Here, as can be seen from the Fig. 3 that starting from different values for radiation, dust and stiff fluid all the situations ultimately approach to the same physical feature.

Finally, we would like to mention that in the present model $\rho$ is positive and hence the Weak Energy Condition (WEC) as well as the Null Energy Condition (NEC) are obeyed here.

\section{Acknowledgments}

The authors (FR \& SR) would like to express their gratitude to the authority of IUCAA, Pune, India for providing them Visiting Associateship under which a part of this work was carried out.
[1] A. G. Riess et al., Astron. J. 116, 1009 (1998)

[2] S. Perlmutter et al., Nat. 391, 51 (1998)

[3] J. D. Barrow and J. Maguijo, astro-ph/9907354 (1999)

[4] E. Garcia-Berrow et al., astro-ph/9907440 (1999).

[5] M. N. C'el'erier, Astron. Astrophys. 353, 63 (2000)

[6] J. M. Overduin and F. I. Cooperstock, Phys. Rev. D 58, 043506 (1998)

[7] V. Sahni and A. Starobinsky, Int. J. Mod. Phys. D 9, 373 (2000)

[8] S. Ray, U. Mukhopadhyay and S. B. Dutta Choudhury, Int. J. Mod. Phys.D 16, 1791 (2007)
[9] R. A. Knop et al., Astrophys. J. 598, 102 (2003)

[10] M. Tegmark et al., Astrophys. J. 606, 702 (2004)

[11] S. V. Chevron and V. M. Zhuravlev, Zh. Eksp. Teor. Fiz. 118, 259 (2000)

[12] V. M. Zhuravlev, Zh. Eksp. Teor. Fiz. 120, 1042 (2001)

[13] P. J. E. Peebles and B. Ratra, Rev. Mod. Phys. 75, 559 (2003)

[14] J. Kujat et al., Astrophys. J. 572, 1 (2002)

[15] M. Bartelmann et al., New Asrton. Rev. 49, 199 (2005)

[16] D. Huterer and M. S. Turner, Phys. Rev. D 64, 123527 (2001) 
[17] J. Weller and A. Albrecht, Phys. Rev. D 65, 103512 (2002)

[18] D. Polarski and M. Chavellier, Int. J. Mod. Phys. 10, 213 (2001)

[19] E. V. Linder, Phys. Rev. Lett. 90, 091301 (2003)

[20] E. V. Linder, Gen. Rel. Gravit. 40, 329 (2008)

[21] D. Rapatti, S. W. Allen and J. Weller, Mon. Not. R. Astron. Soc. 360, 555 (2005)

[22] P.-S. Corasaniti and E. J. Copeland, Phys. Rev. D 67, 063521 (2003)

[23] E. V. Linder and D. Huterer, Phys. Rev. D 72, 043509 (2005)

[24] E. V. Linder in Identification of dark matter (IDM 2002) p. 52, astro-ph/0210217 (2002)

[25] B. Ratra and P. J. E. Peebles, Phys. Rev. D 37, 3406 (1988)

[26] M. S. Turner and M. White, Phys. Rev. D 56, R4439 (1997)

[27] Caldwell et al., Phys. Rev. Lett. 80, 1582 (1998)

[28] A. R. Liddle and R. J. Scherrer, Phys. Rev. D 59, 023509 (1999)

[29] P. J. Steinhardt et al., Phys. Rev. D 59, 123504 (1999)

[30] B. Bhui, B. C. Bhui and F. Rahaman, Astrophys. Space Sc. 299, 61 (2005)

[31] F. Rahaman, B. Bhui and B. C. Bhui, Astrophys. Space. Sc. 301, 47 (2006)

[32] F. Rahaman, M. Kalam and S. Chakraborty, Acta Phys. Polon. B 40, 25 (2009)

[33] L. Amendola, Mon. Not. R. Astron. Soc. 342, 221 (2003)

[34] T. Padmanabhan and T. Roychowdhury, Mon. Not. R. Astron. Soc. 344, 823 (2003)

[35] A. G. Riess, Astrophys. J. 560, 49 (2001)

[36] U. Mukhopadhyay, P. P. Ghosh, M. Khlopov and S. Ray, astro-ph/0711.0686 (2007)

[37] U. Mukhopadhyay, S. Ray and S. B. Dutta Choudhury, Int. J. Mod. Phys. D 17, 301 (2008)

[38] A. A. Usmani, P. P. Ghosh, U. Mukhopadhyay, P. C. Ray and S. Ray, Mon. Not. R. Astron. Soc. 386, L 92 (2008)

[39] U. Mukhopadhyay and S. Ray, N. B. Math. II, 51 (2009)

[40] U. Mukhopadhyay, S. Ray and A. A. Usmani, grqc/0811.0782 (2008)

[41] S. Ray, U. Mukhopadhyay and Xin He-Meng, Gravit. Cosmol. 13, 142 (2007)
[42] F. Gliner, ZETF 49, 542 (1965)

[43] V. Majernik, Phys. Lett. A 282, 362 (2001)

[44] R. G. Vishwakarma, Gen. Relativ. Gravit. 33, 1973 (2001)

[45] I. L. Shapiro, J. Solà and H.S̆tefančić, J. Cosmol. AstroParticle. 01, 012 (2005)

[46] G. V. Vereschagin and G. Yegorian, Class. Quantum Gravit. 23, 5049 (2006)

[47] V. G. Gurzadyan and S. -S. Xue, Mod. Phys.Lett. A 18, $561(2003)$

[48] A. K. Beesham, Phys. Rev. D 48, 8 (1993)

[49] J. C. Carvalho et al., Phys. Rev. D 46, 2404 (1992)

[50] I. Waga, Astrophys. J. 414, 436 (1993)

[51] J. A. S. Lima and J. C. Carvalho, Gen. Relativ. Gravit. 26, 909 (1994) .

[52] R. G. Vishwakarma, Class. Quantum Gravit. 19, 4747 (2002)

[53] A. G. Cohen, D. B. Kaplan and A. E. Nelson, Phys. Rev. Lett. 82, 4971 (1999)

[54] S. D. H. Hsu, Phys. Lett. B 594, 13 (2004)

[55] U. Mukhopadhyay, S. Ray and F. Rahaman, Int. J. Mod. Phys. D in press, (2010)

[56] I. Dymnikova and M. Khlopov, Grav. Cosmol. Suppl. 4, 50 (1998)

[57] I. Dymnikova and M. Khlopov, Mod. Phys. Lett. A 15, 2305 (2000)

[58] I. Dymnikova and M. Khlopov, Eur. Phys. J. C 20, 139 (2001)

[59] U. Mukhopadhyay, P. C. Ray, S. Ray and S. B. Dutta Choudhury, Int. J. Mod. Phys. D 18, 389 (2009)

[60] M. S. Turner, Astrophys. J. 576, L101 (2002)

[61] R. Rebolo, Nucl. Phys. B (Proc. Suppl.) 114, 3 (2003)

[62] J. S. Alcaniz, Phys. Rev. D 69, 083521 (2004)

[63] R. Tripp, Astron. Astrophys. 325, 871 (1997)

[64] V. Sahni, Pramana 53, 937 (1999)

[65] U. Mukhopadhyay and S. Ray, astro-ph/0510557 (2005)

[66] R. R. Caldwell, M. Kamionkowski and N. N. Weinberg, Phys. Rev. Lett. 91, 071301 (2003)

[67] H. S̆tefančić, Phys. Lett. B 595, 9 (2004)

[68] M. Kuhlen et al., Mon. Not. R. Astron. Soc. 357, 387 (2005) 